\title{
Robotic Mapping and Localization Considering Unknown Noise Statistics*
}

\author{
Hamzah AHMAD $^{* *}$ and Toru NAMERIKAWA ${ }^{* * *}$ \\ ** Graduate School of Natural Science and Technology, Kanazawa University \\ Kakuma-Machi, 920-1192, Ishikawa, Japan \\ E-mail: hamzah@scl.ec.t.kanazawa-u.ac.jp \\ *** Department of System Design Engineering, Keio University \\ 3-14-1 Hiyoshi, Kohoku-ku, Yokohama, 223-8522, Japan
}

\begin{abstract}
This paper presents an analysis of $H_{\infty}$ Filter(HF) for Robotics Mapping and Localization with unknown noise statistics. HF which is also known as the minimax filter is proposed in this paper to estimate the robot and landmarks location while robot moves through an unknown environment. Some of the conditions are proposed to ensure that the state covariance in $\mathrm{HF}$ is converging to a steady state value. Furthermore, the analysis of HF convergence for a robot observing landmarks are presented to examine its behavior through the observations. From the experimental results, HF gives a sufficient estimation about the environment. Subsequently, such a result can provide other available estimation methods with the capability to ensure and improved estimation in robotic mapping and localization problem.
\end{abstract}

Key words : Robotic Mapping, Localization, Unknown Noise Statistics

\section{Introduction}

The robotic mapping and localization problem has gained researcher's attention over some past decades. This problem illustrates an application of a mobile robot whose observing an environment and collecting information efficiently while it is moving through the environment. From its observations, the robot then attempts to build a map. In 1980's, Smith and Cheeseman et.al ${ }^{(1)}$ initiated the robotic mapping problem based on the relationship between landmarks and a robot. Since then, a sequential of interesting developments dramatically has evolved its name to Simultaneous Localization and Mapping problem(SLAM $)^{(2)}$. See Fig.1 for the illustration of SLAM problem which consists of a mobile robot observing some landmarks.

SLAM has been applied in a wide range of applications, indoors or outdoors such as satellite, mining, space exploration, rescue and military. The development of SLAM continues in $2 \mathrm{D}^{(3)}$ or $3 \mathrm{D}$ applications ${ }^{(4)(5)}$ and amazingly expanded to home-based robot applications.

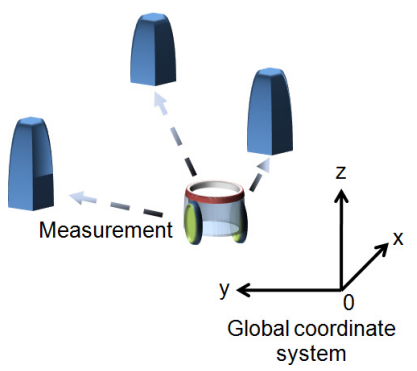

Fig. 1 SLAM problem

*Received 6 Apr., 2010 (№. 10-0148) [DOI: 10.1299/jsdd.5.70]

Copyright $\subset 2011$ by JSME 
SLAM has enhanced from Topological and Metric approaches to Behavioral approach, Mathematical-based model approach and Probabilistic approach ${ }^{(2)}$. However, between these three techniques, the probabilistic approach has made a significant achievement. In spite of probabilistic approach remarkable achievement, such a technique suffers from some shortcomings such as computational complexity. Nevertheless, with modern development of software and research, a considerable support and solution to this problem may exist, thus inspire the development of probabilistic SLAM.

Recently, many approaches using the probabilistic whether parametric or non-parametric methods have been proposed to solve the SLAM problems e.g Kalman Filter, UKF, Particle Filter, etc. Currently, a non-parametric method called Fast-SLAM approach ${ }^{(2)}$, efficiently constructs the unknown map by utilizing an amount of particle whose behaves as the uncertainty to the whole system. If more particles are used, then the estimation result will be better. Unfortunately, this method require higher computational cost. Due to such deficiencies, such a wonderful technique does not deter some classical methods. The readers are encouraged to look at the review about SLAM development explained by S.Thrun et.al ${ }^{(6)}$, which is purposely discussed regarding the SLAM problem from various aspects.

It is a wise decision to model a system that can take into account for the worst case of noises or when the noise statistics are unknown but bounded. Hence, HF can be a complement estimator to tolerate such a robust system. The development of HF for SLAM is proposed herein and its performance has been briefly compared to Kalman Filter ${ }^{(7)(8),(12)-(19)}$ approach in some conditions. $\mathrm{HF}^{(9)}$ is one of the set-membership approaches, which assumed that the noise is known in a bounded energy ${ }^{(10),(11)}$. The energy gain from the noise inputs to the estimation errors is guaranteed to be less than a certain level.

Throughout this paper, we examine the HF about its convergence properties. To guarantee the convergence of state covariance, some conditions are provided. If these conditions are not satisfied, then the estimation have erroneous results. Moreover, we investigate the convergence properties of a robot observing landmarks in an unknown environment. We study the results using a constant motion and sensors uncertainties with a perfect data association considering two cases of gaussian and unknown noise statistics but bounded. To this extent, $H_{\infty}$ Filter is still new in the robotic mapping problem solution schemes, although it has a desirable property and is competitive compared to Kalman Filter. West et.al ${ }^{(12)}$ showed that HF was competent with other well-known approaches such as Kalman Filter and Particle Filter for SLAM problem. However, no theoretical explanation or contribution about HF properties was discussed. In this paper, HF convergence properties are analyzed to understand its behavior in SLAM.

This paper is organized as follows. In Section 2, HF-Based SLAM is presented with a brief comparison to the Kalman Filter, while Section 3 demonstrates the main results of convergence properties of $H_{\infty}$ SLAM problem. Next, Section 4 shows the experimental results of SLAM about both filters performance. Finally, Section 5 concludes the paper.

\section{2. $H_{\infty}$ Filter Based-SLAM}

\subsection{SLAM Mathematical Model}

SLAM consists of two general models which are the process model that explains how the robot move through the unknown environment and the measurement model that calculates and measures the relative distance and angle between robot and landmarks for each respective time. This section analyzes both models to describe how a map can be built using both models information. Fig. 2 explains about these two models. An assumption of stationary landmarks is made for convenience. The process model is presented as follows.

$$
X_{k+1}=f\left(X_{k}, \omega_{k}, v_{k}, \delta \omega_{k}, \delta v_{k}\right), \quad X_{k}=\left[\begin{array}{cccc}
\theta_{k} & x_{k} & y_{k} & L_{k}^{i}
\end{array}\right]^{T}
$$

where $\theta_{k}$ is the robot angle and $x_{k}, y_{k}$ are robot $x, y$ positions. $L_{k}^{i}$ is the $i^{\text {th }}$ landmarks containing its $x_{i}, y_{i}$ positions. Note that we assume the landmarks are stationary and therefore is time invariant unlike the robot location. $v_{k}$ and $\omega_{k}$ are representing the robot velocity and turning 

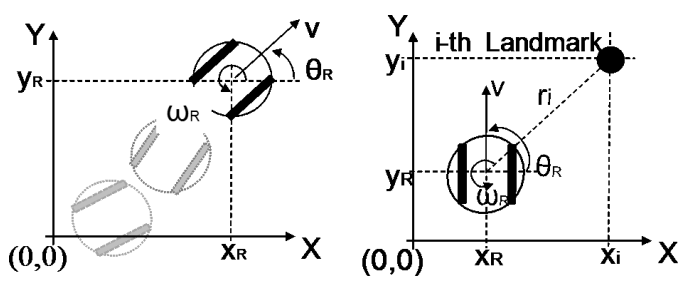

Fig. 2 Process model(left) and measurement model(right) of mobile robot localization

rate respectively. $\delta v_{k}, \delta \omega_{k}$ are the correlated noises for each $v_{k}$, and $\omega_{k} . T$ is the transpose of the matrix. On the other hand, the measurement model has the following equation.

$$
z_{k}=\left[\begin{array}{l}
r_{i} \\
\phi_{i}
\end{array}\right]=\left[\begin{array}{c}
\sqrt{\left(y_{i}-y_{k}\right)^{2}+\left(x_{i}-x_{k}\right)^{2}}+v_{r_{i}} \\
\arctan \left(\frac{y_{i}-y_{k}}{x_{i}-x_{k}}\right)-\theta_{k}+v_{\phi_{i}}
\end{array}\right] \rightarrow \nabla H_{i} X_{k+1}+v_{k}
$$

where $r_{i}, \phi_{i}$ are the relative distance and angle between robot and $i^{\text {th }}$ landmark while other variables hold the same definitions as described before. $v_{r_{i}}$ and $v_{\phi_{i}}$ are the associated noises to the measurements of relative distance and angle respectively. $\nabla H_{i}$ and $v_{k}$ are defined as the measurement model in Jacobian representation and the measurement noise with covariance $R_{k}$, respectively. Both terms are shown by the following notations.

$$
\nabla H_{i}=\left[\begin{array}{ccccc}
0 & -\frac{d x}{r} & -\frac{d y}{r} & \frac{d x}{r} & \frac{d y}{r} \\
-1 & \frac{d y}{r^{2}} & -\frac{d x}{r^{2}} & -\frac{d y}{r^{2}} & \frac{d x}{r^{2}}
\end{array}\right], \quad v_{k}=\left[\begin{array}{c}
v_{r_{i}} \\
v_{\phi_{i}}
\end{array}\right]
$$

where $r=\sqrt{\left(x_{i}-x_{k}\right)^{2}+\left(y_{i}-y_{k}\right)^{2}}, d x=x_{i}-x_{k}$ and $d y=y_{i}-y_{k}$. The prediction step yield the following equations.

$$
\begin{aligned}
& \hat{X}_{k+1}=f\left(\hat{X}_{k}, \omega_{k}, v_{k}, 0,0\right) \\
& P_{k+1}=\nabla f_{X} P_{k} \psi_{k}^{-1} \nabla f_{X}^{T}+\nabla f_{\omega v} \Sigma_{k} \nabla f_{\omega v}^{T}
\end{aligned}
$$

where $\hat{X}_{k}$ is a predicted augmented state and $P_{k}$ is a state error covariance of the system. As shown above, $\Sigma_{k}$ acts as a control noise $\left(\delta \omega_{k}, \delta v_{k}\right)$ covariance.

$$
\nabla f_{X}=\left[\begin{array}{cccc}
1 & 0 & 0 & 0 \\
-v t \sin \theta & 1 & 0 & 0 \\
v t \cos \theta & 0 & 1 & 0 \\
0 & 0 & 0 & I
\end{array}\right], \quad \nabla f_{\omega v}=\left[\begin{array}{c}
\nabla g_{\omega v} \\
0
\end{array}\right]
$$

where $t$ is a sampling time and $I$ is an identity matrix with an appropriate dimension. $\nabla f_{X}$ is the Jacobian transformation of robot motions and $\nabla f_{\omega v}$ is the linearized process noise. We assume no process noise for landmarks. Therefore the linearized process noise for robot motion is $\nabla g_{\omega v} . \psi_{k}$ is shown by the following expression.

$$
\psi_{k}=\left(I+\nabla H_{i} R_{k}^{-1} \nabla H_{i}^{T} P_{k}-\gamma^{-2} I P_{k}\right)
$$

where $\gamma>0$. In $H_{\infty}$ Filter $^{(7)}$, the ratio of estimation error to the noise errors must be less than $\gamma^{2}$. We show this characteristics later. Using the Jacobian notation for a case of a robot observing one landmark at point $A$, we use the following notation.

$$
\nabla H_{A}=\left[\begin{array}{lll}
-e & -A & A
\end{array}\right]
$$

The following abbreviation for Eq.(8) is used and is restated as below.

$$
H_{A}=\left[\begin{array}{ll}
e & A
\end{array}\right], \quad e=\left[\begin{array}{ll}
0 & -1
\end{array}\right]^{T} \Longrightarrow \nabla H_{A}=\left[\begin{array}{ll}
-H_{A} & A
\end{array}\right]
$$

where

$$
A=\left[\begin{array}{ll}
\frac{x_{i}-x_{A}}{\sqrt{x_{i}-x_{A}^{2}+y_{i}-y_{A}^{2}}} & \frac{y_{i}-y_{A}}{\sqrt{x_{i}-x_{A}{ }^{2}+y_{i}-y_{A}^{2}}} \\
\frac{y_{i}-y_{A}}{x_{i}-x_{A}^{2}+y_{i}-y_{A}^{2}} & \frac{x_{i}-x_{A}}{x_{i}-x_{A}^{2}+y_{i}-y_{A}^{2}}
\end{array}\right]
$$

$\left(x_{i}, y_{i}\right)$ and $\left(x_{A}, y_{A}\right)$ are the $i^{t h}$ landmark coordinate and robot position at point A respectively. The initial state covariance $P_{0}>0\left(P_{0} \in \mathbb{R}^{(3+2 N) \times(3+2 N)}\right)$ is presented by

$$
P_{0}=\left[\begin{array}{cc}
P_{0 v} & 0 \\
0 & P_{0 m}
\end{array}\right]
$$

where $P_{0 v}$ is the robot initial covariance and $P_{0 m}$ is the landmarks initial covariance. $N$ is the number of landmarks. 


\section{2. $\boldsymbol{H}_{\infty}$ Filter Properties}

This subsection presents the development of HF-Based SLAM considering about its convergence properties. Its algorithm is also included in some literatures ${ }^{(21),(22)}$. The comparison between $H_{\infty}$ Filter and Kalman Filter convergence ${ }^{(23)}$ for a robot observing landmarks is evaluated based on some experiments. Hamzah et.al ${ }^{(24)-(26)}$ studied the HF convergence and its application with Covariance Inflation to decrease SLAM computation cost. Katayama ${ }^{(7)}$ and D.Simon ${ }^{(9)}$ have presented satisfactory explanations about HF. Referring to them, we first make some assumptions about the noise characteristics.

Assumption 1. $R_{k} \triangleq D_{k} D_{k}^{T}>0$

Assumption 2. Bounded noise energy; $\Sigma_{t=0}^{N}\left\|\zeta_{k}\right\|^{2}<\infty, \Sigma_{t=0}^{N}\left\|\xi_{k}\right\|^{2}<\infty$

From Eq.(1)-(2), we can derive $D_{k}=\left[\begin{array}{ll}0 & I\end{array}\right]$. Assumption 1 defines that the measurement noise are not correlated to process noise during robot observations. Above assumptions are similar to the standard Kalman Filter assumptions where all components of the measurement vectors are assumed to be corrupted by temporally uncorrelated noise, $R_{k}$. Both process noise $\zeta_{k}$ with covariance $Q_{k}$ and the measurement noise $\xi_{k}$ with covariance $R_{k}$ are assumed to be bounded as described by Assumption 2. For details of $H_{\infty}$ Filter, refer to Katayama $^{(7)}$ and Lewis et.al ${ }^{(21)}$.

The differences between Kalman Filter and $H_{\infty}$ Filter appears in its form of gain, $K_{k}$ and its state covariance $P_{k}$, which integrates both the prediction and updates process. For Kalman Filter, the equation for its gain and covariance are given by,

$$
\begin{aligned}
& K_{k}=P_{k}\left(I+\nabla H_{i}^{T} R_{k}^{-1} \nabla H_{i} P_{k}\right)^{-1} \\
& P_{k+1}=\nabla F_{k} P_{k}\left(I+\nabla H_{i}^{T} R_{k}^{-1} \nabla H_{i} P_{k}\right)^{-1} \nabla F_{k}^{T}+Q_{k}
\end{aligned}
$$

On the other hand, $H_{\infty}$ Filter gain and covariance are expressed by Eqs.(14)-(15).

$$
\begin{aligned}
& K_{k}=P_{k}\left(I-\gamma^{-2} P_{k}+\nabla H_{i}^{T} R_{k}^{-1} \nabla H_{i} P_{k}\right)^{-1} \\
& P_{k+1}=\nabla F_{k} P_{k}\left(I-\gamma^{-2} P_{k}+\nabla H_{i}^{T} R_{k}^{-1} \nabla H_{i} P_{k}\right)^{-1} \nabla F_{k}^{T}+Q_{k}
\end{aligned}
$$

where $\gamma$ is an $H_{\infty}$ norm of the closed-loop system. $H_{\infty}$ Filter depends on the covariance matrix of error signals, $Q_{k} \geq 0, R_{k}>0$ which are chosen and designed such that to achieve a desired performance. It is observable that if $\gamma$ becomes bigger in (14) and (15), then these equation are the same as (12), (13) of Kalman Filter.

An $H_{\infty}$ Filtering problem is defined as following. Given that for $P_{0}>0\left(P_{0} \in \mathbb{R}^{3+2 N}\right)$, the state covariance yields

$$
\begin{aligned}
& P_{k+1}=\nabla f_{X} P_{k} \psi_{k}^{-1} \nabla f_{X}^{T}+\nabla f_{\omega v} \Sigma_{k} \nabla f_{\omega v}^{T}, \quad P_{0}=\Sigma_{0} \\
& \psi_{k}=I+\left(\nabla H_{i}^{T} R_{k}^{-1} \nabla H_{i}-\gamma^{-2} L_{k}^{T} L_{k}\right) P_{k}
\end{aligned}
$$

In our case $L_{k}$ is an identity matrix with an appropriate dimension. The above equations holds a positive definite solution if it satisfies the following equation.

$$
P_{k}=\hat{P}_{k}^{-1}-\gamma^{-2} L_{k}^{T} L_{k}>0, \quad k=0,1, \ldots, N
$$

where

$$
\hat{P}_{k}=\left(P_{k}^{-1}+\nabla H_{i}^{T} R_{k}^{-1} \nabla H_{i}\right)^{-1}>0
$$

For $\gamma>0$, the suboptimal HF is given by below expressions.

$$
\begin{aligned}
& \hat{c}_{k}^{*}=L_{k} \hat{x}_{k}, \quad \hat{x}_{k+1}^{-}=\nabla F_{k} \hat{x}_{k} \\
& \hat{x}_{k+1}^{+}=\hat{x}_{k}+K_{k}\left[z_{k}-\nabla H_{i} \hat{x}_{k}\right], \hat{x}_{0}=\bar{x}_{0} \\
& K_{k}=P_{k} \nabla H_{i}\left(\nabla H_{i} P_{k} \nabla H_{i}^{T}+R_{k}\right)^{-1}
\end{aligned}
$$

where $\hat{c}_{k}^{*}$ is the interested state output estimation, $\hat{x}_{k+1}^{-}$and $\hat{x}_{k+1}^{+}$are the priori and posteriori estimated state, and $\bar{X}_{0}$ is the estimated initial state with its associated initial state covariance, $P_{0}$. In other words, given $\gamma>0$, an $H_{\infty}$ Filter attempts to find a solution for $\hat{x}_{k}$ that satisfies the following equation.

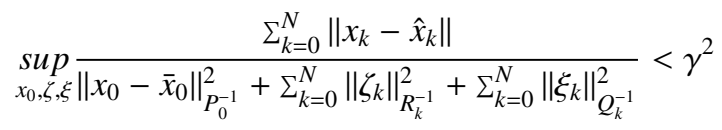




\section{Main Results}

To obtain a successful estimation using $H_{\infty}$ Filter, a solution to the $H_{\infty}$ Filter must be available $\mathrm{e}^{(22)}$. If else, a faulty estimations and finite escape time problem is observable. The finite escape time states that an estimation suddenly goes to infinity in finite time horizon.

Assumption 3. $\left(\nabla F_{k}, \nabla H_{i}\right)$ is observable and $\left(\nabla F_{k}, \nabla G_{k}\right)$ is controllable.

From Eqs.(1)-(2), we can derive $\nabla G_{k}=\left[\begin{array}{ll}I & 0\end{array}\right]$. This setting is suggested to guarantee that the process noise is uncorrelated to the measurement noise. With respect to Calleja et.al[8], we present the following lemma for the stability of $H_{\infty}$ Filter. Note that from now on we change $\nabla H_{i}$ to $H_{i}$ unless stated.

Lemma 1. The filtering error dynamics for an HF is converging to a zero mean steady state if and only if the matrix $I-K_{k} H_{i}$ is stable and Eq.(18) is satisfied. $H_{i}$ and $K_{k}$ are defined by Eq.(3) and Eq.(22) respectively.

Proof. We begin the proof by showing that $\left(I-K_{k} H_{i}\right)$ is marginally stable in robotic mapping problem. Given that the initial state covariance matrix given by,

$$
P_{0}=\left[\begin{array}{ccc}
\sigma_{\theta}^{2} & 0 & 0 \\
0 & \sigma_{x y}^{2} * I_{2} & 0 \\
0 & 0 & \sigma_{m}^{2} * I_{2 N}
\end{array}\right]
$$

where $\sigma_{\theta}, \sigma_{x y}$, and $\sigma_{m}$ are the robot pose angle, robot $x_{k}, y_{k}$ positions and landmarks $x_{i}, y_{i}$ initial covariance respectively. $I$ is an identity matrix with an appropriate dimension. For a stationary robot whose observing a landmark, the measurement model yields

$$
H_{i}=\left[\begin{array}{l}
r_{i} \\
\phi_{i}
\end{array}\right]=\left[\begin{array}{ccc}
0 & -1 & 1 \\
-1 & 0 & 0
\end{array}\right]
$$

If Eq.(19) is satisfied, and by using Eq.(22) about the filter gain, then the following equation is derived as

$$
\begin{aligned}
K_{k} & =P_{0} H_{i}^{T}\left(H_{i} P_{k} H_{i}^{T}+R_{k}\right)^{-1} \\
& =\left[\begin{array}{cc}
0 & -\sigma_{\theta}^{2} s_{\theta} \\
-\left(\sigma_{x y}^{2} * I_{2}\right) s & 0 \\
\left(\sigma_{m}^{2} * I_{2 N}\right) s & 0
\end{array}\right]
\end{aligned}
$$

where $s, s_{\theta}$ are the innovation variances and $s=\left(\left(\sigma_{x y}^{2} * I_{2}\right)+\left(\sigma_{m}^{2} * I_{2 N}\right)+R_{k}\right)^{-1}, s_{\theta}=\left(\sigma_{\theta}^{2}+R_{k}\right)^{-1}$. Hence,

$$
\begin{aligned}
& \left(I-K_{k} H_{i}\right) \\
& =\left[\begin{array}{ccc}
I-\sigma_{\theta}^{2} s_{\theta} & 0 & 0 \\
0 & I_{2}-\left(\sigma_{x y}^{2} * I_{2}\right) s & \sigma_{x y}^{2} s \\
0 & -\sigma_{m}^{2} s & I_{2 N}-\left(\sigma_{m}^{2} * I_{2 N}\right) s
\end{array}\right]
\end{aligned}
$$

The eigenvalues of the above matrix is obtained as

$$
\left[1-\frac{\sigma_{\theta v m}}{2} \mp \frac{1}{2}\left[\sigma_{\theta m}^{2}-\sigma_{\theta}\left(\sigma_{x y}^{2} * I_{2}\right)-\sigma_{\theta}\left(\sigma_{m}^{2} * I_{2 N}\right)\right]^{\frac{1}{2}}\right]
$$

where $\sigma_{\theta v m}=\sigma_{\theta}^{2}+\left(\sigma_{x y}^{2} * I_{2}\right)+\left(\sigma_{m}^{2} * I_{2 N}\right)$ and $s, s_{\theta} \neq 0$. Shown above, there exist at least an eigenvalue leading to partial stability and there are two other solutions for each sign of the second term. In addition, $\left(I-K_{k} H_{i}\right)$ secure that there exist a steady state solution for the Differential Riccati Equation(DRE).

Lewis et.al ${ }^{(21)}$ guaranteed that if $I-K_{k} H_{i}$ is stable, then the DRE of HF is converging. This result shows the same structure to Calleja et.al ${ }^{(8)}$ analysis. We also found that most of the results from Dissayanake et.al ${ }^{(3)}$ in linear cases are applicable for HF. These are due to the preceding explanations of both filters stated in Eqs.(12)-(15). The system gain and covariance characteristics explicitly shows HF behavior towards SLAM. To determine this, we investigate 
its properties especially about its the convergence characteristics. From this point forward, we present those results consisting about HF convergence properties.

Theorem 1. Assumes that Assumptions $1 \sim 3$ are satisfied. For $\gamma>0$, the predicted state error of a robot observing landmarks is gradually decreasing if observations are frequently made by a robot. Moreover, this is achieved if and only if $R_{k}<\gamma^{2}$.

Proof. This property is analyze using Eqs.(18)-(19). Consider a linear case SLAM problem. Let $\nabla f_{X}=I$ and the observation noise covariance, $R_{k}>0$. As the initial state covariance is $P_{0}=P_{0}^{T}>0$, from both equations, it shows that if $R_{k}<\gamma^{2}$, then $H_{i}^{T} R_{k}^{-1} H_{i}-\gamma^{-2} I \geq 0$. Hence,

$$
\begin{aligned}
& P_{k}=\left(P_{0}^{-1}+H_{i}^{T} R_{k}^{-1} H_{i}-\gamma^{-2} I\right)^{-1} \leq P_{0} \\
& P_{k+1}=\left(P_{k}^{-1}+H_{i}^{T} R_{k}^{-1} H_{i}-\gamma^{-2} I\right)^{-1} \leq P_{k}
\end{aligned}
$$

If the robot observes a landmark for some time, then the updated Eq.(29) simultaneously become smaller and therefore result in nearer estimation to the true value. From the positive semidefinite(PsD) properties, it is known that the map state covariance $P_{k_{m m}}$ also exhibits the same properties.

$$
\begin{aligned}
& P_{k_{m m}}=\left(P_{0_{m m}}^{-1} k+H_{i}^{T} R_{k}^{-1} H_{i}-\gamma^{-2} I\right)^{-1} \leq P_{0_{m m}} \\
& P_{k+1_{m m}}=\left(P_{k_{m m}}^{-1}+H_{i}^{T} R_{k}^{-1} H_{i}-\gamma^{-2} I\right)^{-1} \leq P_{k_{m m}}
\end{aligned}
$$

Subsequently, the map state covariance is also decreasing. Therefore, as presented above, the whole state covariance matrix becomes smaller after a sufficient observations if and only if, $H_{i}^{T} R_{k}^{-1} H_{i}-\gamma^{-2} I \geq 0$. This condition plays an important role in HF -SLAM.

Theorem 1 had demonstrated that the whole state covariance matrix is decreasing if it is satisfying $H_{i}^{T} R_{k}^{-1} H_{i}-\gamma^{-2} I \geq 0$. This expression describes its importance in HF estimation. Bolzern et.al ${ }^{(22)}$ suggested some requirements to guarantee HF convergence in some conditions. In Theorem $6^{(22)}$, HF is converging only if for some given positive scalar $\varepsilon$ such that the initial state covariance $P_{0}$ satisfies the following.

$0 \leq P_{0} \leq P^{s}+(Y+\varepsilon I)^{-1}$

where $Y=Y^{T}$ is the solution of Lyapunov function and $P^{s}$ is the stabilizing solution. However, technically it is difficult to determine $\varepsilon$. Even more, this equation equivalently means that if no solution is available, then we should increase $\gamma$. By increasing $\gamma$, a solution is guaranteed to exist as it closer to EKF behavior. Besides that, by calculating $P^{s}$ online, the equation leads to computational cost. As a result designer must first compute the stabilizing solution with consideration of the noises parameters, initial state covariance while at the same guaranteeing the ratio of estimation error to the noises distribution is less than $\gamma$. Thereby, we suggest very general requirements to apply HF-based SLAM which require perceiving only the behavior of updates via Eq.(28) and satisfying $R_{k}<\gamma^{2}$ in the system ${ }^{(26)}$.

Corollary 1. Given that $P_{0}=P_{0}^{T}>0$. For each observation, if $R_{k}>\gamma^{2} I$, the whole state covariance becomes a negative definite matrix or indefinite and lead to unstable estimations.

Proof. Assume that in each observation, $H_{i}^{T} R_{k}^{-1} H_{i}-\gamma^{-2} I<0$. For $P_{0}>0$, especially in a case when the robot is stationary, then for $R_{k}>\gamma^{2}$, the following can be obtained.

$$
\begin{aligned}
& P_{k}=\left(P_{0}^{-1} k+H_{i}^{T} R_{k}^{-1} H_{i}-\gamma^{-2} I\right)^{-1}<0 \\
& P_{k+1}=\left(P_{k}^{-1}+H_{i}^{T} R_{k}^{-1} H_{i}-\gamma^{-2} I\right)^{-1}>P_{k}
\end{aligned}
$$

Subsequently, the whole state covariance matrix becomes a negative definite matrix and continues to increase to negative value that is unacceptable behavior in SLAM.

Corollary 1 generally verifies that the estimation may turn out to be insufficient if Theorem 1 is not satisfied and become worst if $P_{0}>>0$ as stated in Eq.(32). Besides, Corollary 1 explains that HF requires the designer to carefully choose the observation noise 
and some other parameters. Moreover, $\gamma$ must be tuned properly to ensure that a PsD matrix is preserved in each robot observation updates. Finite Escape Time is one of the problems of HF. To avoid this problem, some conditions are proposed as follows.

Theorem 2. There exists a solution to HF-SLAM problem if and only if the followings are achieved.

- $\quad$ The observation noise, $R_{k}<<\gamma^{2} I$.

- $\quad$ Theorem 1 is satisfied.

- State covariance matrix is always a PsD in each observation.

If else, the estimation is unreliable and exhibits Finite Escape Time phenomena.

Proof. To examine these conditions, the HF-SLAM algorithm in Section 2.2 is referred. The observation noise must satisfied $R_{k}<\gamma^{2} I$, as this will guarantee that Eq.(18) exhibit a PsD matrix. To demonstrate this, we consider a uni-dimensional robot case which is a robot with single position information observing a single landmark coordinate to show that the solution for HF-SLAM is exist. From Eq.(17), for $P_{0}>0$ and $\gamma^{2}>R_{k}$, we obtained the following equations.

$$
\begin{aligned}
\psi_{k} & =I+\left[\begin{array}{c}
-1 \\
1
\end{array}\right]\left[R_{k}^{-1}\right]\left[\begin{array}{ll}
-1 & 1
\end{array}\right]-\left[\begin{array}{cc}
\gamma^{-2} & 0 \\
0 & \gamma^{-2}
\end{array}\right] P_{0} \\
& =\left[\begin{array}{cc}
1+\left(R_{k}^{-1}-\gamma^{-2}\right) P_{v v} & -R_{k}^{-1} P_{m m} \\
-R_{k}^{-1} P_{v v} & 1+\left(R_{k}^{-1}-\gamma^{-2}\right) P_{m m}
\end{array}\right]
\end{aligned}
$$

$P_{v v}$ and $P_{m m}$ are the robot and landmarks state covariance respectively. From the properties of a PsD matrix, a determinant of its matrix is non-negative. Therefore, it is sufficient to show that Eq.(35) determinant is non-negative. The determinant of Eq.(35) yields

$$
\psi_{k}=P_{v v} P_{m m}+\left[2\left(R_{k}^{-1}-\gamma^{-2}\right)-2 R_{k}^{-1} \gamma^{-2}+\gamma^{-4}\right] P_{v v} P_{m m}
$$

To ease calculation, choose $\gamma^{2}>>R_{k}$. Then we obtained that

$$
\psi_{k} \rightarrow P_{v v} P_{m m}+2 R_{k}^{-1} P_{v v} P_{m m} \geq 0
$$

As a result, $\psi_{k}$ is always a PsD. Hence, the linearized state covariance yield

$$
\begin{aligned}
& \psi_{k}=I+\left(H_{i}^{T} R_{k}^{-1} H_{i}-\gamma^{-2} I\right) P_{k} \geq 0 \\
& P_{k+1}=F_{k} P_{k} \psi_{k}^{-1} F_{k}+G Q_{k} G^{T} \geq 0
\end{aligned}
$$

Note that, $\gamma^{2} I>>R_{k}$ is a general condition that must be selected to achieve a reliable estimation. If else, it exhibits a non PsD matrix. Theorem 1 has shown that if $H_{i}^{T} R_{k}^{-1} H_{i}-\gamma^{-2} I \geq 0$, then the state covariance is decreasing. Finally the state covariance matrix is converging to some steady state value. If Eq.(17) exhibits a non PsD matrix, then the estimation becomes inaccurate as shown by Corollary 1 . This is also a sign of finite escape time.

We now moves to investigate the convergence behavior of HF when a robot is observing some landmarks. It is often useful to examine the state covariance matrix since it stands for the whole uncertainties characteristics about a system. S.Huang et.al ${ }^{(3),(23)}$ have analyzed Kalman Filter-Based SLAM convergence properties. In HF, the convergence properties of a robot observing landmarks are still unavailable. This is due to HF is a new approach in SLAM and a very few researchers have applied the filter as a solution to the problem. Hence we obtained the following theorem to understand the HF estimation behavior in SLAM problem especially in unknown noise statistics but bounded.

Theorem 3. For a stationary robot observing a stationary $i^{\text {th }}$ landmark, we assume more than $n$-times $(n>0)$ observations are made. For a given $\gamma>0$, if $n$ goes to infinity, the whole covariance matrix is converging to

$$
P_{m}^{\infty} \rightarrow\left[\begin{array}{ll}
0 & 0 \\
0 & 0
\end{array}\right]
$$


Proof. 2D robot with initial covariance matrix $P_{0}$ is considered to examine the above theorem and $P_{0}$ is given by the following,

$$
P_{0}=\left[\begin{array}{cc}
\sigma_{v}^{2} & 0 \\
0 & \sigma_{m}^{2}
\end{array}\right]
$$

where $\sigma_{v} \in \mathbb{R}^{3}$ is the robot initial state covariance and $\sigma_{m} \in \mathbb{R}^{2 i}$ is the landmark initial state covariance. Assume that the stationary robot is observing one landmark $i$. From Eq.(17), when the robot is observing $i$ landmark $n$ times, and if $R_{k}=\sigma_{r}^{2} I$, then we obtain the following. Here, $I$ is an identity matrix with an appropriate dimension.

$$
\begin{aligned}
\psi_{k} & =I+n\left(H_{i}^{T} R_{k}^{-1} H_{i}-\gamma^{-2} L_{k}^{T} L_{k}\right) P_{k} \\
& =I+n\left[\begin{array}{cc}
R_{k}^{-1}-\gamma^{-2} I & R_{k}^{-1} \\
R_{k}^{-1} & R^{-1_{k}}-\gamma^{-2} I
\end{array}\right]\left[\begin{array}{cc}
\sigma_{v_{k}}^{2} & \sigma_{v m_{k}} \\
\sigma_{m v_{k}} & \sigma_{m_{k}}^{2}
\end{array}\right] \\
& =\left[\begin{array}{ll}
\rho_{11} & \rho_{12} \\
\rho_{21} & \rho_{22}
\end{array}\right]
\end{aligned}
$$

where each element in Eq.(42) is shown by

$$
\begin{array}{ll}
\rho_{11}=I+n\left(R_{k}^{-1}-\gamma^{-2}\right) \sigma_{v_{k}}^{2}, & \rho_{12}=\left(n R_{k}^{-1}\right) \sigma_{m_{k}}^{2} \\
\rho_{21}=\left(n R_{k}^{-1}\right) \sigma_{v_{k}}^{2}, & \rho_{22}=I+n\left(R_{k}^{-1}-\gamma^{-2}\right) \sigma_{m_{k}}^{2}
\end{array}
$$

Finding the inverse matrix of Eq.(42) by the Matrix Inversion Lemma yields

$$
\psi_{k}^{-1}=\left[\begin{array}{ll}
\psi_{11} & \psi_{12} \\
\psi_{21} & \psi_{22}
\end{array}\right]
$$

where

$$
\begin{aligned}
& \psi_{11}=\left[1+n\left(R_{k}^{-1}-\gamma^{-2}\right) \sigma_{v}^{2}-n R_{k}^{-1} \sigma_{m}^{2} \times\left(1+n\left(R_{k}^{-1}-\gamma^{-2}\right)\right)^{-1} R_{k}^{-1} \sigma_{v}^{2}\right]^{-1} \\
& \psi_{12}=-\psi_{11} n R_{k}^{-1} \sigma_{m}^{2}\left[1+n\left(R_{k}^{-1}-\gamma^{-2}\right)\right]^{-1} \\
& \psi_{21}=\psi_{12}^{T} \\
& \psi_{22}=\left[1+n\left(R_{k}^{-1}-\gamma^{-2}\right)\right]^{-1}+\left[1+n\left(R_{k}^{-1}-\gamma^{-2}\right)\right]^{-1} \times n R_{k}^{-1} \sigma_{m}^{2} \psi_{11} n R_{k}^{-1} \sigma_{m}^{2}\left[1+n\left(R_{k}^{-1}-\gamma^{-2}\right)\right]^{-1}
\end{aligned}
$$

As $n \rightarrow \infty$ and for convenience assume that the correlation terms are very small and therefore, can be neglected. It then yields

$$
\psi_{\infty}^{-1}=\left[\begin{array}{ll}
0 & 0 \\
0 & 0
\end{array}\right]
$$

Substituting Eq.(44) into Eq.(16), finally we obtain that if $n$-observations are made, the state covariance $P^{\infty}$ becomes as

$$
\begin{aligned}
P^{\infty} & =P_{k} \psi_{\infty}^{-1} \\
& =\left[\begin{array}{ll}
0 & 0 \\
0 & 0
\end{array}\right]
\end{aligned}
$$

It is recognizable that in the limit, the whole state covariance matrix is decreasing. We discovered that, the designer must satisfy Theorem 1, Theorem 2. Otherwise, the results may not be expected. Although above result encourages good estimation for the robot and landmark positions, the true landmark location is still unknown ${ }^{(23)}$. Even more, similar to Kalman Filter, if bigger magnitude of noises are applied to the filter, then oscillations becomes slightly bigger. Hence it may give an impact to the overall estimations.

In normal application, the robot has to observe more than a landmark to increase its confidence about its surroundings. We further study the convergence properties when the non-moving robot was observing two stationary landmarks. Let $\sigma_{v}$ be robot state covariance and $\sigma_{m}$ as landmark state covariance. Besides, assume that $\sigma_{v m}=\sigma_{m v}, \sigma_{v \bar{m}}=\sigma_{\bar{m} v}$ as the correlation between robot and landmarks. Then the following rule is propose.

Proposition 1. For a stationary robot observing two stationary landmarks, $m$ and $\bar{m}$ with $\gamma>0$, as more $n$ times observations are made, the whole covariance matrix is converging to 


$$
P_{m}^{\infty}=\left[\begin{array}{ccc}
\sigma_{v}^{2} & \sigma_{v} \sigma_{m} & \sigma_{v} \sigma_{\bar{m}} \\
\sigma_{m} \sigma_{v} & \sigma_{m}^{2} & \sigma_{m} \sigma_{\bar{m}} \\
\sigma_{\bar{m}} \sigma_{v} & \sigma_{\bar{m}} \sigma_{m} & \sigma_{\bar{m}}^{2}
\end{array}\right]
$$

In the limit, the covariance matrix finally becomes

$$
P_{m}^{\infty}=\left[\begin{array}{lll}
0 & 0 & 0 \\
0 & 0 & 0 \\
0 & 0 & 0
\end{array}\right]
$$

Proof. The proof is similar to the proof of Theorem 3 and it is omitted.

These results are extendable only for a single robot observing a number of landmarks. Note that, the map covariance for landmark 1 and landmark 2 are different and varied to each other, especially whenever the initial covariance matrix is not equal to zero and if and only if the uncertainties are differ from each other. As a conclusion, the uncertainties of those two landmarks are differ to each other and therefore contributes about of its dependence to the initial covariance.

\section{Experimental Results}

\subsection{Stationary robot observing landmarks}

Experiments have been conducted by using an e-puck robot to observe some point landmarks in order to evaluate above proposed analysis. We assume that the landmarks are not occluded to each other and are available in sensor coverage. The process noise is assumed to be very small than the measurement noise and both noises are able to represent our environment conditions. Table 1 shows the experimental parameters.

Table 1 EXPERIMENTAL PARAMETERS

\begin{tabular}{|c|c|}
\hline Process noise distribution, $\mathrm{Q}$ & $1 \times 10^{-7} * \operatorname{diag}\left(I_{3}\right)$ \\
\hline Observation noise distribution, $\mathrm{R}$ & 0.05 (for each $\left.r_{i}, \theta_{i}\right)$ \\
\hline Observation noise(uniform noise), $\mathrm{R}$ & {$\left[\begin{array}{c}R_{\theta_{\max }}=0.1 \\
R_{\theta_{\min }}=-0.08 \\
R_{\text {distance }_{\max }}=0.3 \\
R_{\text {distance }_{\min }}=0.1\end{array}\right]$} \\
\hline Initial covariance, & $P_{v v}=1 \times 10^{-5} * I_{3}$ \\
$P_{0}$ (stationary) & $P_{m m}=1 \times 10^{5} * I_{2 m}$ \\
\hline Initial covariance, & $P_{v v}=6 \times 10^{-3} * I_{3}$ \\
$P_{0}$ (moving) & $P_{m m}=6 \times 10^{-2} * I_{2 m}$ \\
\hline$\gamma($ stationary) & 0.9 \\
\hline$\gamma($ moving) & 8 \\
\hline Sampling time & $0.1 \mathrm{~s}$ \\
\hline
\end{tabular}

For a stationary robot case, two landmarks are defined at two positions at $(50,0)$ and $(60,0)$ in millimeters $(\mathrm{mm})$ respectively while the robot is at $(0,0)$. The landmarks are assume as point landmarks with respect to a global coordinate system. See Fig.3 that presents the experiment environment. From Fig.4, it is easy to identify that the HF estimates very well for its position and landmarks. From the sensor's measurements, HF performs well in both landmark inference with reliable convergence results and thus proves Theorem 3. Besides that, no finite escape time phenomena is perceived during experiment. As the robot is stationary at $(0,0)$, Fig.4(a) equivalently means that the estimation error is also decreasing and approximating the true value of $(0,0)$ even though the associated figure is not mentioned here. Note that, HF estimation converges faster than KF especially for the landmarks estimations. The same characteristics can be interpreted from Fig.4(b) for the landmarks estimation error where both landmark 1 and 2 estimation error are monotonically decreasing as more observations are made.

On the other hand, when $\gamma<<R$, HF performance is incapable of achieving better results than Kalman Filter(see Fig.5). This result is caused by ineffective estimation about robot localization and landmark's estimation. Consequently, this proves Theorem 1 and Theorem 2 


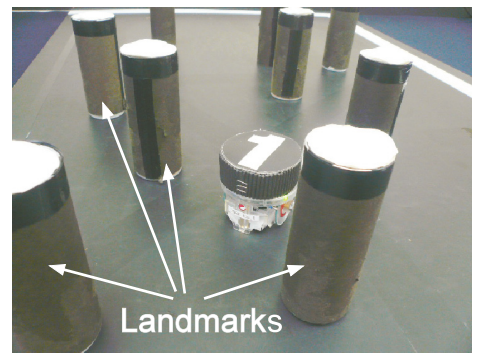

Fig. 3 Stationary Epuck observing landmarks

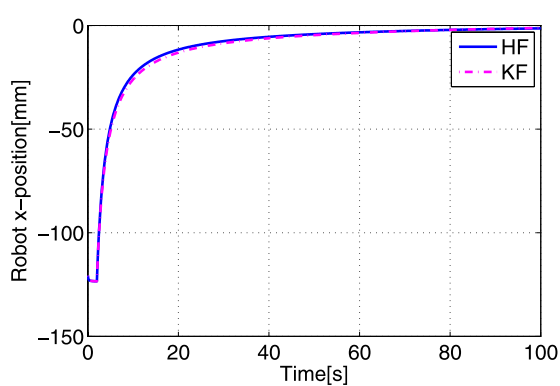

Fig.4(a) Robot position estimation
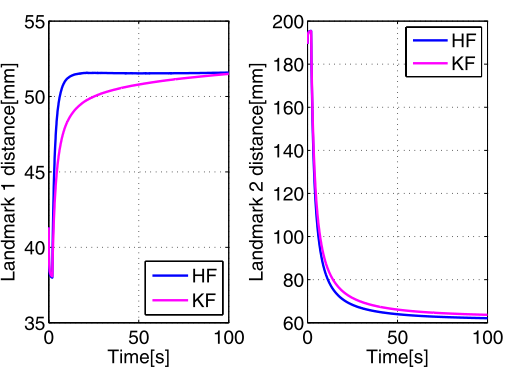

Fig.4(b) Landmark 1 estimation

Fig. 4 HF estimation about robot and landmarks position

when some conditions are not satisfied. The situation also synonymously means that the estimation errors are keep increasing during observations. In linear case, this is happened especially when the initial state covariance $P_{0}>>0$ and if (28) or (29) exhibits a negative definite matrix where $R_{k}^{-1}-\gamma^{-2} I<0$. Therefore, this situation result in $P_{0}^{-1}+R_{k}^{-1}-\gamma^{-2} I<0^{(26)}$. Remark that commonly in SLAM, the initial state covariance holds large value as the robot does not know where it is located in the environment before performing any given tasks.

\subsection{Moving robot observing landmarks}

The experiment for a case of moving robot observing landmarks is conducted to evaluate the consistency of HF in SLAM in both gaussian and non-gaussian noises characteristics. Figs.6-7 demonstrates that $\mathrm{HF}$ is more robust especially in robot pose angle estimation than EKF. Even if the resulted covariance is slightly higher than EKF state covariance, HF has the best solution for the respective case. These results also agrees with preceding findings regarding the state covariance update(See Bolzern et.al ${ }^{(22)}$ and Eqs.(12)-(15) for better comprehension). Furthermore, these results shows that HF converges faster than EKF about the position and consistent to our previous results ${ }^{(24)}$. The EKF robot path estimation is become inconsistent especially when robot turns(See Fig.6(a) and Fig.7). Subsequently, the landmarks estimation becomes faulty due to EKF inconsistent estimation. Remark that robot angle is important to achieve better performance in $\operatorname{SLAM}^{(23)}$. A slight difference in angle estimation leads to different estimation for the landmarks positions.

Considering uniform noise statistic, HF still surpassed EKF estimations(again refer to Fig.7 when robot turns). The HF robot path estimation gives better result than EKF with better robot pose angle estimation. Observes the figure at the first time when robot turns and when robot finished estimating its surroundings. In addition, in an experiment when the robot initial covariance is $1 \times 10^{2}$ while landmarks initial covariance is $1 \times 10^{4}$, HF again shows good performance with $\gamma=12$ (see Fig.8). In most of the observations, HF estimation surpasses EKF estimation. At the beginning of estimation EKF shows big error while HF estimation keeps near to the true value for both robot path and landmarks locations. Thus, these results 

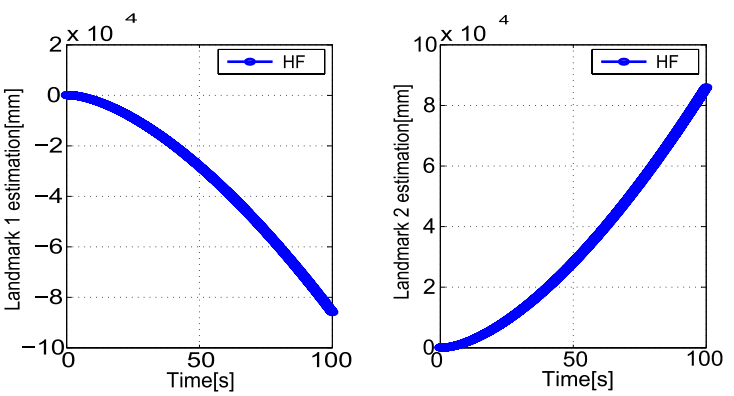

Fig. 5 Landmark estimation: Effect when $R>>\gamma^{2}$

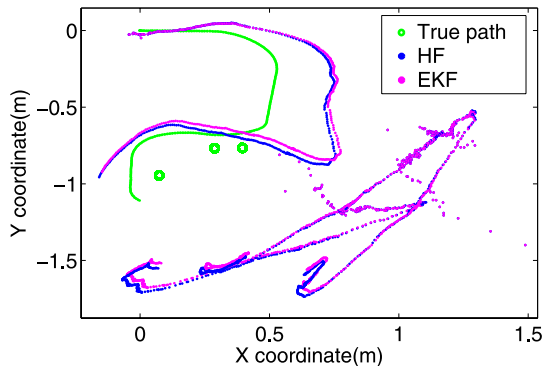

Fig.6(a) Biased gaussian noise
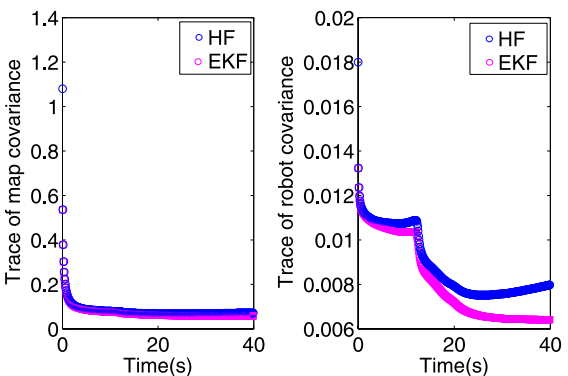

Fig.6(b) State Covariance behavior

Fig. 6 Built map under HF Estimation under biased gaussian noise and its associated state covariance

inspires further development for $\mathrm{HF}$ while at the same time guaranteeing $\mathrm{HF}$ as a solution to the SLAM problem.

\subsection{Discussion}

The results have shown that HF is very useful and competent to EKF performance especially in a case of non-Gaussian noise characteristics. Instead of using Particle Filter(PF), which suffers from computational cost and requires larger data storage, HF can be a best candidate for SLAM problem as it holds almost the same characteristics to Kalman Filter with lower computational cost and memory storage. Furthermore, in our best knowledge, PF-based SLAM still is incapable to be employed successfully online. Thus, the implementation of HF in SLAM is a very considerable approach. Nevertheless, its performance in a case of an environment with unknown noise characteristics is a notable achievement, which enable a simpler approach than the PF.

\section{Conclusions}

It has been shown that HF is able to be a complement filter for unknown noise statistics in SLAM. Besides that, HF is applicable in linear and non-linear system that has variety of noises and useful for SLAM problem. We demonstrated that state covariance of HF is converging if the robot keeps observing any landmarks. However, there are some circumstances to be considered to ensure that the estimation results achieved the expected performance. If these conditions are ignored, then the estimation result in erroneous estimation and probably exhibits Finite Escape Time problem which is undesirable situation in SLAM. In addition, the experimental results showed that HF is a good selection for an environment with unknown noise statistics. Even though it seems that the estimation is almost the same to Kalman Filter, HF improved better about the robot angle estimation which is very important in SLAM as stated by S.Huang et.al. However, designer should design an appropriate level of weighting noise $Q_{k}$, and $R_{k}$ to achieve a certain level of performance in HF. In addition, by applying the 


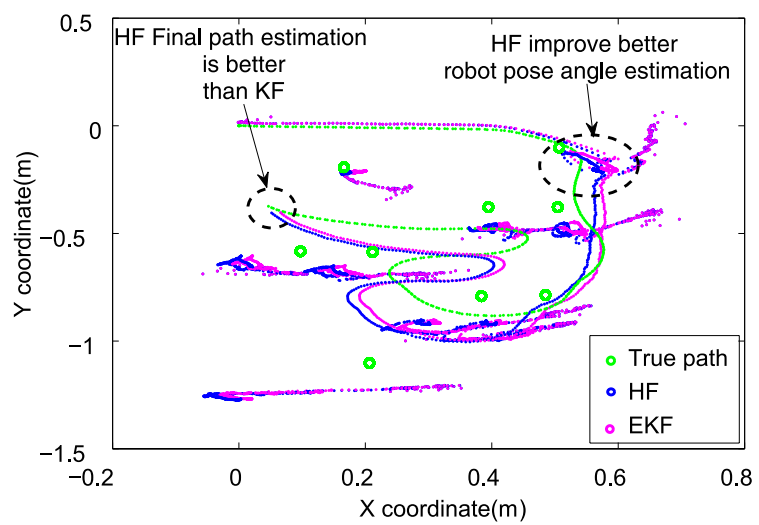

Fig. 7 HF and EKF Filter built map under uniform noise characteristics

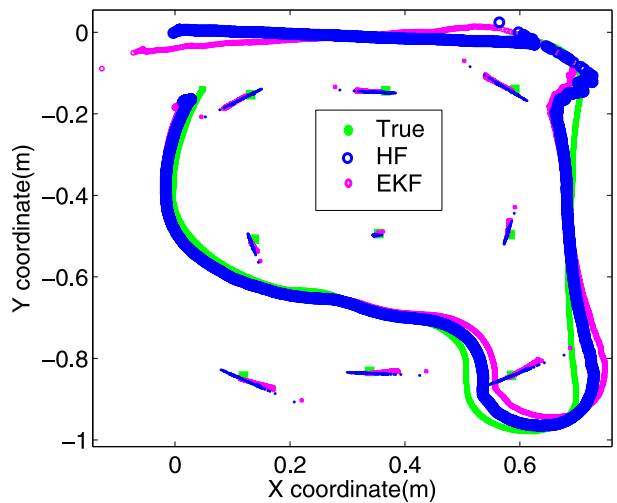

Fig. 8 Estimation with bigger initial covariance

HF-SLAM, the computation cost is preserved and better than the PF approach which suffers from computational cost and difficult to apply in real-time SLAM.

\section{Acknowledgements}

The authors would like to thanks Kementerian Pengajian Tinggi Malaysia, the University Malaysia Pahang, Mr.Yusuke Morimoto and Mr.Ohtani Tatsuya for their continuous supports.

\section{References}

( 1 ) R. C. Smith and P. Cheeseman, On the representation and estimation of spatial uncertainty. Technical Report TR 4760 and 7239, SRI, (1985).

( 2 ) S. Thrun, W. Burgard, D. Fox, Probabilistic Robotics, MIT Press, (2005).

( 3 ) G. Dissayanake, P. Newman, S. Clark, H. Durrant-Whyte, M. Csorba, A Solution to the Simultaneous Localization and Map Building(SLAM) problem, IEEE Transaction of Robot and Automation, Vol. 17, No. 3, (2001), pp.229-241.

( 4 ) S. Thrun, W. Burgard, D. Fox, A Real-Time Algorithm for Mobile Robot Mapping with Applications to Multi-robot and 3d Mapping, In Proc. IEEE Intl. Conf. on Robotics and Automation, Vol.1, (2000), pp.321-328.

( 5 ) D. Hahnel, W. Burgard, and S. Thrun, Learning Compact 3d Models of Indoor and Outdoor Environments with a Mobile Robot, In Proc. European workshop on Advanced Mobile Robots, (2001).

(6) S. Thrun, Robotic mapping, A survey, Exploring Artificial Intelligence in the New Millenium, G. Lakemeyer and B. Nebel, Eds.Morgan Kaufmann, (2003), pp.1-35.

( 7 ) T. Katayama, Applied Kalman Filter, Asakura Shoten, (2000), pp.191-211. 
( 8 ) T. Vidal-Calleja, J. Andrade-Cetto, A. Sanfeliu, Conditions for Suboptimal Filter Stability in SLAM, Proceedings of 2004 IEEE/RSJ International Conference on Intelligent Robots and Systems, (2004), pp.27-32.

( 9 ) D. Simon, From Here to Infinity, Embedded Systems Programming, Vol.14, No.11, (2001), pp.20-32.

(10) A. Sabater, F. Thomas, Set Membership Approach to the Propagation of Uncertain Geometric Information, Proceedings of 1991 IEEE International Conference on Robotics and Automation, (1991), pp.2718-2723.

(11) A. Garulli, A. Vicino, Set Membership Localization of Mobile Robots via Angle Measurements, IEEE Transaction on Robotics and Automation, Vol.17 No.4, (2001), pp.450463.

(12) M. E. West and V. L. Syrmos, Navigation of an autonomous underwater vehicle (AUV) using robust SLAM, Proc. 2006 IEEE CCA, (2006), pp.1801-1806.

(13) L. M. Paz, J. Neira, Optimal local map size for EKF-based SLAM, IEEE/RSJ International Conference of Intelligent Robots and Systems, (2006), pp.5019-5025.

(14) S. Rongchuan, M. Shugen, L. Bin, W. Yuechao, Improving consistency of EKF-based SLAM algorithms by using accurate linear approximation, IEEE/ASME International Conference of Advanced Intelligent Mechatronics, (2008), pp.619-624.

(15) R. Martinez-Cantin, J. A Castellanos, Bounding Uncertainty in EKF-SLAM: The Robocentric Local Approach, IEEE Proceedings of International Conference of Robotics and Automation, (2006), pp.430-435.

(16) T. Bailey, J. Nieto, J. Guivant, M. Stevens, E. Nebot, Consistency of the EKF-SLAM Algorithm, IEEE/RSJ International Conference of Intelligent Robots and Systems, (2006), pp.3562-3568.

(17) J. A. Castellanos, J. M. M. Montiel, J. Neira, and J. D. Tardos, The SPmap: A Probabilistic Framework for Simultaneous Localization and Map building, IEEE Transaction of Robot and Automation, Vol.15, No.5, (1999), pp.948-953.

(18) J. Leonard, H. Durrant-Whyte, and I. Cox, Dynamic map building for an autonomous mobile robot, The International Journal of Robotics Research, Vol.11, No.4, (1992), pp.286-298.

(19) S. Thrun, Y. Liu, D. Koller, A. Y. Ng, Z. Ghahramani, and H. Durrant-Whyte, Simultaneous Localization and Mapping with Sparse Extended Information Filters, The International Journal of Robotics Research, Vol.23, No.7-8, (2004), pp. 693-716.

(20) R. Smith, M. Self, P. Cheeseman, Estimating Uncertain Spatial Relationship in Robotics, in Autonomous Robot Vehicles, I.J.Cox and G.T Wilfon, New York Springer Verlag, (1990), pp.167-193.

(21) F. L. Lewis, L. Xie, D. Popa, Optimal and Robust Estimation, 2nd. Ed. CRC Press, (2008), pp.353-410.

(22) P. Bolzern, P. Colaneri, G. De Nicolao, $H_{\infty}$-differential Riccati equations: Convergence Properties and Finite Escape Phenomena, IEEE Transactions of Automatic Control, Vol.42, No. 1, (1997), pp.113-118.

(23) S. Huang, G. Dissayanake, Convergence and consistency Analysis for Extended Kalman Filter Based SLAM", IEEE Transaction on Robotics, Vol.23, No.5, (2007), pp.10361049.

(24) H. Ahmad, T. Namerikawa, $H_{\infty}$ Filter Convergence and Its Application to SLAM, ICROS-SICE International Joint Conference, (2009), pp.2875-2880.

(25) H. Ahmad, T. Namerikawa, Feasibility Study of Partial Observability in $H_{\infty}$ Filtering for Robot Localization and Mapping Problem, American Control Conference, (2010), pp.3980-3985.

(26) H.Ahmad, T.Namerikawa, Robot Localization and Mapping Problem with Unknown Noise Characteristics, 2010 IEEE Multi-conference on Systems and Control, Yokohama, Japan, (2010), pp.1275-1280. 